\title{
Hydrogen Diffusion Simulation in Dual Phase Microstructure Considering the Difference in Diffusion Constants*
}

\author{
by Gen Ogita**, Yoshiki Mikami*** and Masahito Mochizuki***
}

\begin{abstract}
To evaluate the effect of the dual-phase microstructure on hydrogen diffusion behavior, a numerical simulation using a two-dimensional dual-phase model was performed. The effect of the dual-phase microstructure was evaluated by changing the morphological parameters such as the shape and geometrical distribution of the austenite phase using two different phase fractions. The results indicated that the hydrogen does not diffuse through the higher coefficient phase on a preferential basis; instead, the diffusion occurs with the same time dependency in both phases. In addition, the layered pattern of the austenitic phase and the higher phase fraction of the austenite phase reduced the degree of hydrogen diffusion. The hydrogen diffusion in dual-phase steel is significantly affected by the difference in the hydrogen diffusion constants and morphology of the microstructure of the dual-phase steel.
\end{abstract}

Key Words: Hydrogen Diffusion, Dual-phase Steel, Numerical Simulation, Microstructure, Coefficient

\section{Introduction}

Recently, high strength steel and high corrosion resistance steel have been widely used in gas and oil production and in refining units that require weight saving or longer life cycle. Many of these steels have a dual-phase microstructure, including X65, $\mathrm{X} 80$, and duplex stainless steels. However, high strength steel has a higher sensitivity to hydrogen-related cracking such as hydrogen-induced cracking in service, including hydrogen- and cold-cracking of welding joints. Therefore, the hydrogen diffusion behavior in steel has been extensively studied ${ }^{1)-6)}$; however, the effect of the dual-phase microstructure on this behavior has not yet been well explained.

To predict the hydrogen diffusion and distribution in steel, a numerical simulation has been applied because the hydrogen diffusion is difficult to observe experimentally. Hydrogen diffusion simulations have been performed in the past decades; however, the effects of the microstructure and morphology on the hydrogen diffusion have remained unclear. In particular, the hydrogen distribution in the dual-phase microstructure consisting of different hydrogen diffusion constants such as diffusion coefficients and solubilites is difficult to infer experimentally.

A hydrogen diffusion simulation has been performed using a finite element simulation model consisting of a dual-phase microstructure of ferrite and martensite phases. The hydrogen diffusion coefficients and mechanical properties of ferrite and martensite are different, and the difference significantly affects the hydrogen concentration distribution and stress concentration distribution on the microscopic scale ${ }^{7)}$. However the influence of

\footnotetext{
${ }^{*}$ Received: 2014.11 .28

${ }^{* *}$ Non-member, Chiyoda Corporation.

${ }^{* * *}$ Member, Division of Materials and Manufacturing Science, Graduate School of Engineering, Osaka University
}

morphology of dual phased microstructure has not been discussed.

In this study, in order to evaluate the effect of dual phase microstructure, a numerical simulation using the two-dimensional dual-phase model was performed. Dual-phase microstructure is consisted with ferrite and austenite, which has the different diffusion constants. The effect of the dual-phase microstructure on the hydrogen diffusion behavior was evaluated by changing the morphology of the microstructure, the diffusion coefficients and solubilites. The effect of phase fraction on hydrogen diffusion behavior is also discussed.

\section{Numerical Simulation Method of Microscopic Hydrogen Diffusion}

\subsection{Governing Equation}

The numerical simulation of hydrogen diffusion on the microscopic scale was performed based on the generalized Fick's diffusion law described in Eqs. (1) and (2) ${ }^{9), 10)}$.

$$
\begin{aligned}
& \mathbf{J}=-s \mathbf{D}\left\{\frac{\partial \phi}{\partial \mathbf{x}}+\kappa_{s} \frac{\partial}{\partial \mathbf{x}}\left(\ln \left(\theta-\theta^{Z}\right)\right)+\kappa_{p} \frac{\partial p}{\partial \mathbf{x}}\right\} \\
& \int_{V} \frac{d c}{d t} d V+\int_{S} \mathbf{n} \cdot \mathbf{J} d S=0
\end{aligned}
$$

where

$$
\begin{aligned}
& s(\theta, \mathbf{f}): \text { solubility }(\mathrm{ppm}) \\
& \mathbf{D}(c, \theta, \mathbf{f}) \text { : diffusion coefficient }\left(\mathrm{mm}^{2} / \mathrm{s}\right) \\
& \kappa_{\mathrm{s}}(c, \theta, \mathbf{f}) \text { : Soret effect factor } \\
& \kappa_{\mathrm{p}}(c, \theta, \mathbf{f}) \text { : equivalent pressure gradient effect factor } \\
& V_{H} \text { : partial molar volume of hydrogen in the solid solution } \\
& \quad\left(=2 \times 10^{-6} \mathrm{~m}^{3} / \mathrm{mol}\right)
\end{aligned}
$$




\author{
$R$ : gas constant $(=8.3143 \mathrm{~J} / \mathrm{K} \cdot \mathrm{mol})$ \\ $\theta$ : temperature $(\mathrm{K})$ \\ $\theta_{\mathrm{Z}}$ : absolute zero temperature $(\mathrm{K})$ \\ $\varphi$ : normalized concentration $(=c / s)$ \\ $c$ : concentration (ppm) \\ $p$ : equivalent pressure stress $(=-\operatorname{trace}(\sigma) / 3, \mathrm{MPa})$ \\ f: the number of microstructure \\ $V$ : arbitrary volume $\left(\mathrm{mm}^{3}\right)$ \\ $S$ : surface of $\mathrm{V}\left(\mathrm{mm}^{2}\right)$ \\ n: normal vector of $S$ \\ $\mathbf{J}$ : flux of concentration $(\mathrm{ppm} \cdot \mathrm{mm} / \mathrm{s})$.
}

The driving force of hydrogen diffusion is the generalized chemical potential considering the normalized concentration gradient, temperature gradient, and equivalent pressure gradient and is summarized on the right side of the Eq. (1). Based on the above formulation, the microscopic hydrogen distribution was calculated. The simulation was performed using the commercial finite element software Abaqus.

\subsection{Hydrogen Diffusion Simulation Model}

To evaluate the effect of the dual-phase microstructure, a numerical simulation with a two-dimensional dual-phase model was performed. Dual-phase models with ferrite and austenite were generated to investigate the effect of the microstructure on the hydrogen diffusion behavior. It is suitable to investigate the effect of the dual-phase microstructure because the hydrogen diffusion coefficients between austenite and ferrite are large compared with other dual-phase combinations. The effect of the dual-phase morphology on the overall diffusion behavior of the model is discussed by computing the hydrogen diffusion behavior using the simulation models.

The model used for the numerical simulation is shown in Fig. 1. A hydrogen concentration of $1 \mathrm{ppm}$ was used for Edge A of the simulation model as a boundary condition. The model size was

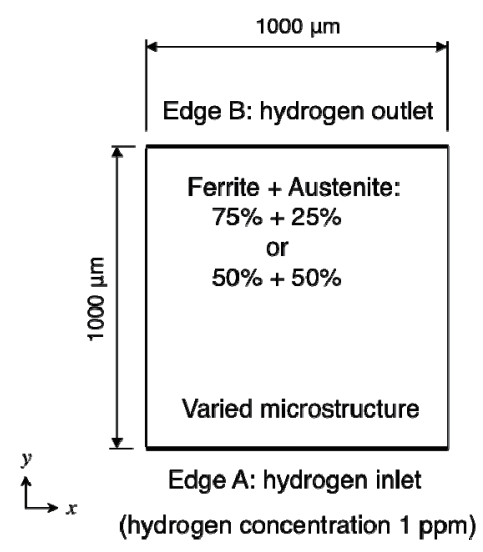

Fig. 1 Numerical simulation model
$1000 \mu \mathrm{m} \times 1000 \mu \mathrm{m}$; the hydrogen inlet was set on the bottom edge (Edge A), and the hydrogen outlet was set on the upper edge (Edge B). The hydrogen diffusion coefficients used in the simulation were $6.0 \times 10^{-6} \mathrm{~mm}^{2} / \mathrm{s}$ for ferrite and $3.3 \times 10^{-10} \mathrm{~mm}^{2} / \mathrm{s}$ for austenite ${ }^{8)}$. The solubility of the ferrite and austenite phases were set to $2.8 \times 10^{-2} \mathrm{ppm}$ and $70 \mathrm{ppm}$, respectively at $293 \mathrm{~K}^{8}$. Only hydrogen diffusion in the vertical direction from Edge A to B is discussed in this paper, and no hydrogen was emitted outside of the model from other model edges. The total calculation time for the hydrogen diffusion simulation was $1.00 \times 10^{9}$ seconds.

To determine the effect of the morphological distribution of the two phases, two patterns of the distribution of the austenite phase in the ferrite matrix were used, as illustrated in Fig. 2. In addition, two different phase fractions were applied to each patterned model: $25 \%$ austenite and $75 \%$ ferrite, and $50 \%$ austenite and $50 \%$ ferrite. For the $25 \%$ austenite phase fraction, the distribution of the austenite phase was set to random and layered as shown in models (a-1) and (a-2), and for the 50\% austenite phase fraction, the distribution of the austenite phase was set to random and layered as shown in models (b-1) and (b-2). Dual-phased models were generated based on the morphology in the micrograph of the cross section for duplex stainless steel. In the random pattern, the single austenite phase was sized $10 \mu \mathrm{m} \times 10 \mu \mathrm{m}$ and distributed randomly. In the layered pattern, which resembled the

Gray: Ferrite $(\alpha)$, Black: Austenite $(\gamma)$

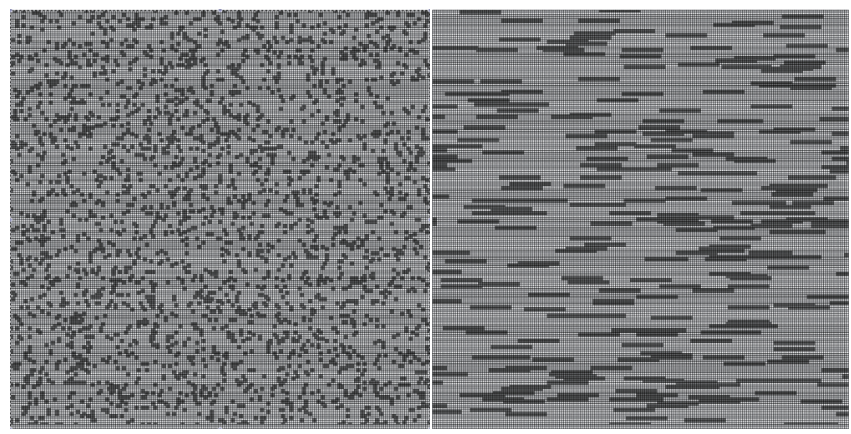

(a-1) random $25 \% \gamma$

(a-2) layered $25 \% \gamma$

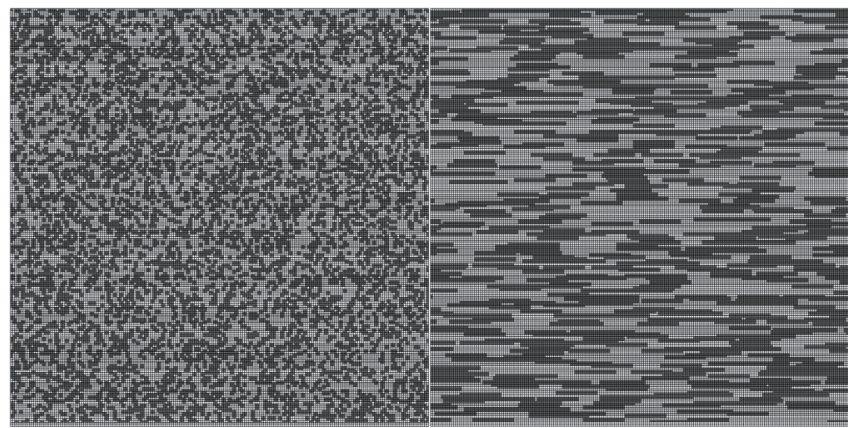

(b-1) random $50 \% \gamma$

(b-2) layered $50 \% \gamma$

Fig. 2 Microstructures of the dual-phase models 
morphology of a rolled steel plate microstructure in the cross section of the longitudinal plane cut in the thickness direction, single austenite phase was sized $10 \mu \mathrm{m} \times 100 \mu \mathrm{m}$ and patterned randomly.

\section{Results and Discussion}

\subsection{Hydrogen Distribution for Different Microstructures}

The simulation results are discussed using the time ratio to the total calculation time $\left(=t / t_{\text {total }}\right)$ hereinafter. The results of the hydrogen diffusion simulation after approximately 0.03 of total calculation time $\left(=t_{1}\right)$ for the four patterns are presented in Fig. 3 , and the calculation results after approximately 0.18 of total calculation time $\left(=t_{2}\right)$ are presented in Fig. 4. Comparing the results in models (a-1) and (a-2) in Fig. 4, the hydrogen concentration at Edge B (upper edge) reaches $70 \mathrm{ppm}$ in the austenite phase of model (a-1), which is equivalent to the solubility of austenite, indicating saturation. The concentration remains at $50 \mathrm{ppm}$ in the austenite phase of model (a-2) and is not yet saturated. Despite the same ferrite-austenite phase fraction being set for the two models, the hydrogen distributions are microscopically different in each model. In the (a-2) model in

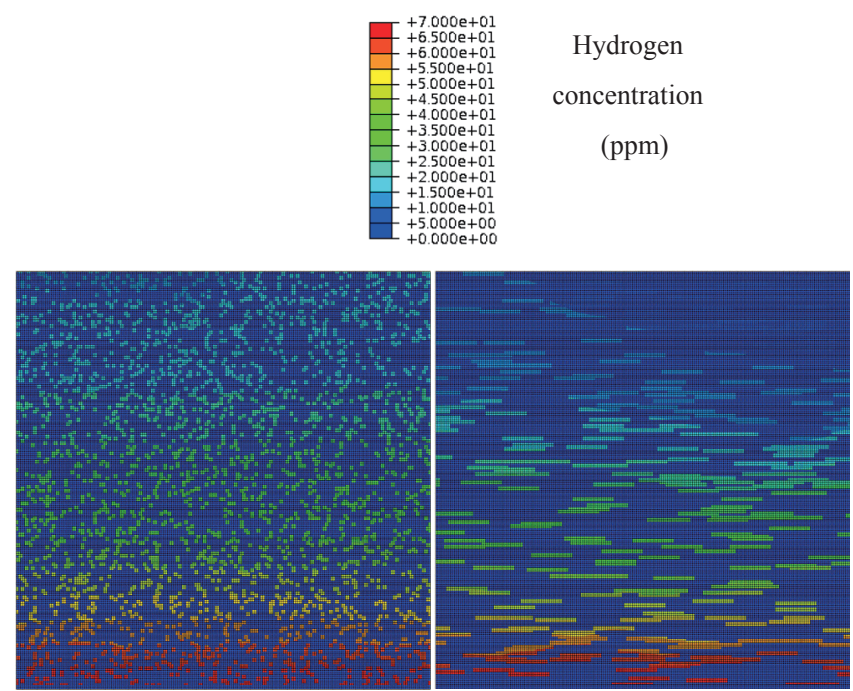

(a-1) random $25 \% \gamma$

(a-2) layered $25 \% \gamma$
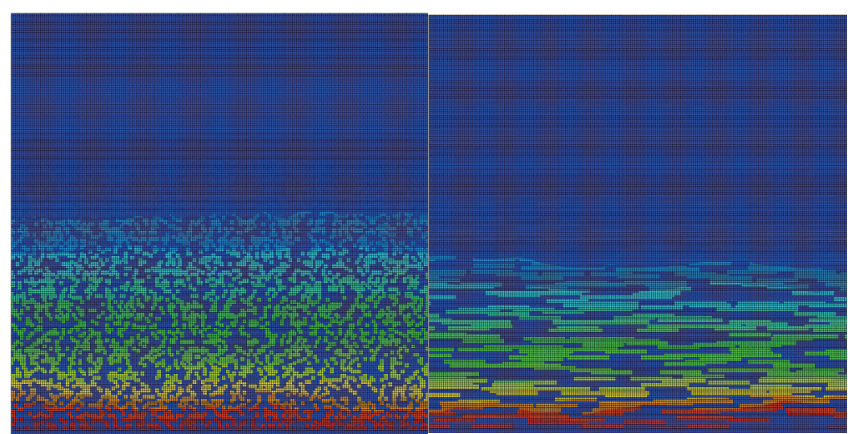

(b-1) random $50 \% \gamma$

(b-2) layered $50 \% \gamma$

Fig. 3 Hydrogen concentration distribution near $t_{1}$.
Figs. 3 and 4, the hydrogen diffusion is obstructed by the layered austenite phases. The same trend is observed in the models set with ferrite-austenite phase fractions of $50 \%$ and $50 \%$ in models (b-1) and (b-2). Comparing the results of the models containing the same aspect austenite phase with different ferrite-austenite phase fractions in models (a-1) and (b-1) in Fig. 3, the hydrogen concentration level in model (b-1) in the austenite phases at the center of the model is approximately 10 to $15 \mathrm{ppm}$. Compared with the hydrogen concentration level in the austenite phases at the same time and location in model (a-1), the hydrogen level is approximately 35 to $40 \mathrm{ppm}$, which is double the concentration level in model (b-1). The phase fraction is observed to affect the hydrogen diffusion rate in the dual-phase microstructure.

\subsection{History of Hydrogen Concentration in the Model}

Figures 5 and 6 present the hydrogen concentration in the austenite phase and ferrite phase, respectively, with the time history for each model. The hydrogen concentration value is the average concentration values in each austenite phase and ferrite phase at Edge $\mathrm{B}$ and was used to evaluate the overall diffusion behavior of each model quantitatively. The hydrogen

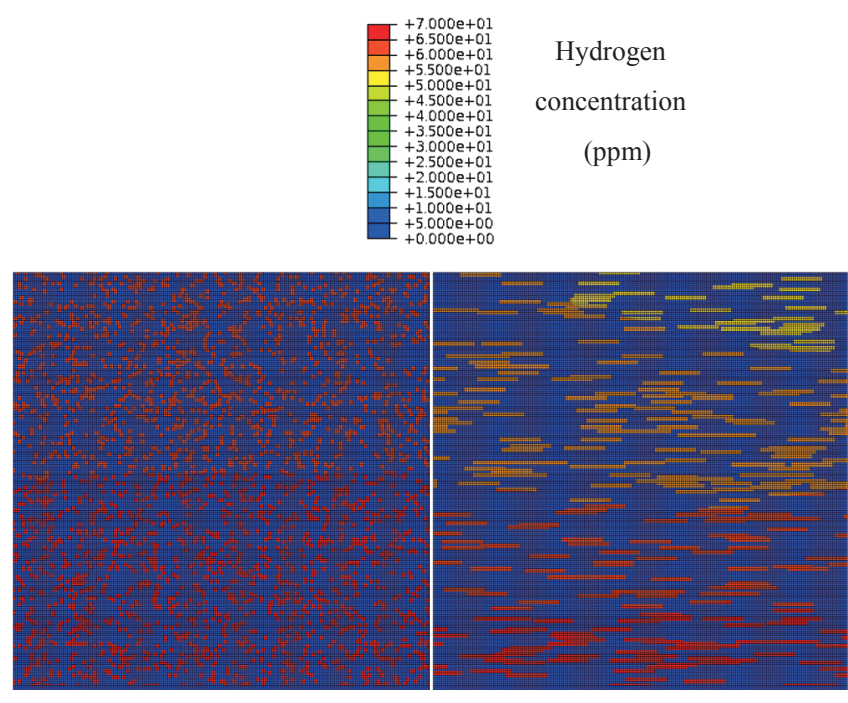

(a-1) random $25 \% \gamma$

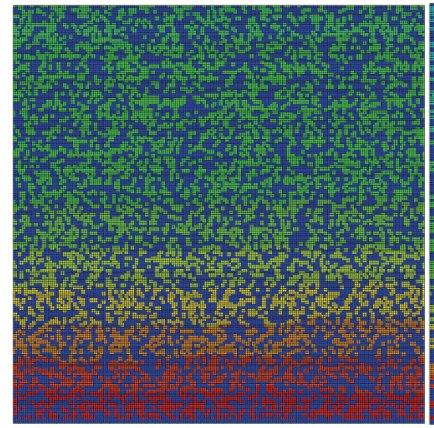

(b-1) random $50 \% \gamma$ (a-2) layered $25 \% \gamma$

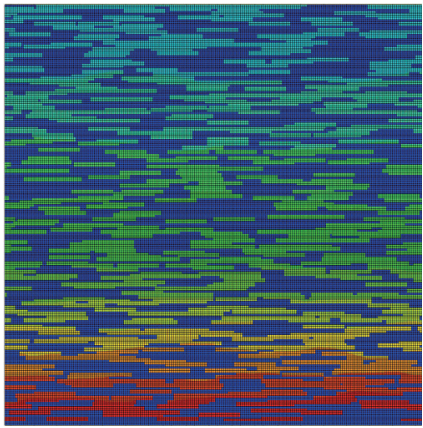

(b-2) layered $50 \% \gamma$
Fig. 4 Hydrogen concentration distribution near $t_{2}$. 
concentration of each model is saturated at $70 \mathrm{ppm}$ in austenite and $2.8 \times 10^{-2} \mathrm{ppm}$ in ferrite, and the saturated value is defined by the given solubility. The hydrogen concentration values at $t_{1}$ and $t_{2}$ are plotted in Figs. 5 and 6 with " $\boldsymbol{\Delta}$ " for $t_{1}$ and "口" for $t_{2}$; the exact calculation times are slightly different in the four models.

First, the hydrogen concentration at Edge $\mathrm{B}$ in between the random patterned models with two different phase fractions, $25 \%$ and 50\% austenite for model (a-1) and model (b-1) are compared. At $t_{1}$ (= the time after approximately 0.03 of total calculation time), the hydrogen concentration in austenite is $15 \mathrm{ppm}$ in model (a-1) and $0.98 \mathrm{ppm}$ in model (b-1), and the concentration ratio is 15. The hydrogen concentration at $t_{2}$ for model (a-1) is $63 \mathrm{ppm}$ and $29 \mathrm{ppm}$ in model (b-1). The model with higher austenite fraction has a lower hydrogen diffusion rate.

Next, comparing the hydrogen concentration level in between model (a-1) and model (a-2), with the same 25\% austenite phase fraction, at $t_{1}$ (= the time after approximately 0.03 of total calculation time), the hydrogen concentration in austenite is 10.4 ppm in the layered model and $14.9 \mathrm{ppm}$ in the random model. The model with the layered shape austenite has a lower hydrogen diffusion rate. Comparing the layered and random pattern models with $50 \%$ austenite, as illustrated in Fig. 5, the hydrogen concentration in the layered model is lower than that in the random model. An effect of the shape of the austenite phase is also observed in the different phase fraction models. Therefore, the cause of the difference is considered to be that the hydrogen diffusion from Edge A to Edge B was obstructed by the layered austenitic phases elongated perpendicular to the diffusion direction. In addition, the difference in the hydrogen concentration between the layered and random models increases in the higher austenite phase fraction models. The effect of the morphology of the microstructure contributes more to reduce the hydrogen diffusion. It can be easily understood that greater obstruction was caused by the elongated austenite phase lying ahead of the hydrogen diffusion direction because of the higher austenite fraction.

Finally, the appearances of all the curvatures in Fig. 5 and Fig. 6 for the four models are very similar, which indicates that the time dependency of the hydrogen diffusion in the austenite phase and ferrite phase is the same. The hydrogen concentration at Edge $\mathrm{B}$ in austenite and ferrite are very different. The concentration ratio between austenite and ferrite is very similar to the ratio of solubility of the austenite and ferrite phases.

\section{Conclusions}

In this study, a two-dimensional numerical simulation method

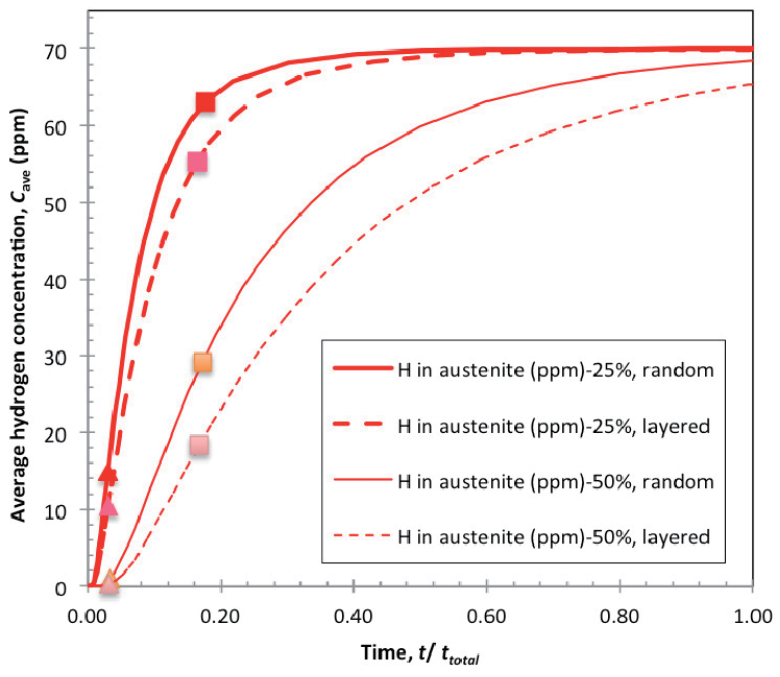

Fig. 5 Hydrogen concentration-time curve at Edge B in austenite phase

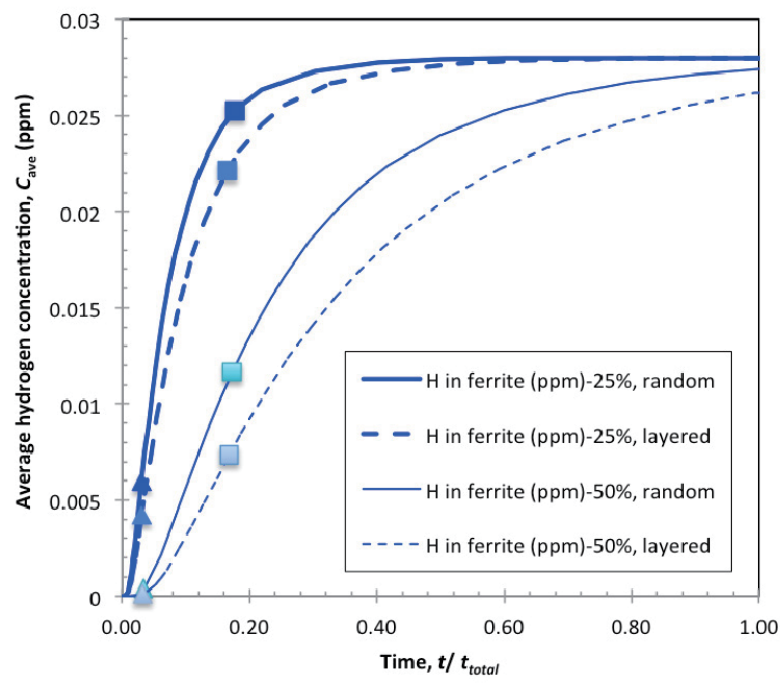

Fig. 6 Hydrogen concentration-time curve at Edge B in ferrite phase

was used to evaluate hydrogen diffusion in various dual-phase microstructures with different material properties. Using the four different microstructural models, the hydrogen distribution and diffusion rate were calculated. Based on the simulation results, the following conclusions were drawn:

1. The hydrogen diffusion rate was affected by the austenite phase fraction. A higher austenite phase fraction results in a lower hydrogen diffusion rate.

2. The apparent hydrogen diffusion coefficients were affected by the difference in the microstructural morphology even if the same fraction of ferrite and austenite phases was applied.

A hydrogen diffusion simulation model with dual-phase microstructure was established. Using this model, the difference in the hydrogen behavior in each phase must be observed under a 
stress-strain field, which is another factor to determine the effect on the behavior.

\section{References}

1) V. Olden, C. Thaulow, R.Johnsen, E. Ostby, T. Berstad, Influence of hydrogen from cathodic protection on the fracture susceptibility of $25 \% \mathrm{Cr}$ duplex stainless steel: Constant load SENT testing and FE-modeling using hydrogen influenced cohesive zone elements, Engineering Fracture Mechanics, 76 (2009), 827-844.

2) E. Owczarek, T. Zakroczymski, Hydrogen transport in a duplex stainless steel, Acta Materialia, 48 (2000), 3059-3070.

3) T. Neeraj, R. Srinivasan, J. Li, Hydrogen embrittlement of ferritic steels, Acta Materialia, 60 (2012), 5160-5171.

4) R. Francis, G. Byme, G.R. Warburton, Effects of cathodic protection on duplex stainless steels in seawater, Corrosion, 53-3 (1997), 234-240.
5) S.L. Chou, W.T. Tsai, Effect of grain size on the hydrogen-assisted cracking in duplex stainless steels, Materials Science and Engineering, A270 (1999), 219-224.

6) W.C. Luu, P.W. Lie, J.K. Wu, Hydrogen transport and degradation of commercial duplex stainless steel, Corrosion Science, 44 (2002), 1783-1791.

7) Y. Mikami, N. Kubota, M. Mochizuki, Numerical simulation of diffusible hydrogen distribution in austenite-martensite microstructure Proceedings of Visual-JW 2012 (2012), 115-116.

8) A. Turnbull, R.B. Hutchings, Analysis of hydrogen atom transport in a two-phase alloy, Materials Science and Engineering, A177 (1994), 161-171.

9) P. Sofronis, R.M. McMeeking, Numerical analysis of hydrogen transport near a blunting crack tip, Journal of Mechanics and Physics of Solids, 37 (1989), 317-350.

10) Dassault Systems Abaqus 6.13 Theory Manual, (2013), 2.13-1-1-4. 